\title{
Le débat sur le travail fondé sur la subsidiarité : un outil pour développer un environnement capacitant
}

The structured debate on work as an approach to developing an enabling

environment

\section{Raoni Rocha, Vanina Mollo et François Daniellou}

\section{(2) OpenEdition}

Journals

Édition électronique

URL : http://journals.openedition.org/activites/2999

DOI : 10.4000/activites.2999

ISSN : 1765-2723

Éditeur

ARPACT - Association Recherches et Pratiques sur les ACTivités

\section{Référence électronique}

Raoni Rocha, Vanina Mollo et François Daniellou, «Le débat sur le travail fondé sur la subsidiarité : un outil pour développer un environnement capacitant », Activités [En ligne], 14-2 | 2017, mis en ligne le 15 octobre 2017, consulté le 30 avril 2019. URL : http://journals.openedition.org/activites/2999; DOI : 10.4000/activites.2999

Ce document a été généré automatiquement le 30 avril 2019.

\section{cc) () $\Theta$}

Activités est mis à disposition selon les termes de la licence Creative Commons Attribution - Pas d'Utilisation Commerciale - Pas de Modification 4.0 International. 


\title{
Le débat sur le travail fondé sur la subsidiarité : un outil pour développer un environnement capacitant
}

\author{
The structured debate on work as an approach to developing an enabling \\ environment
}

Raoni Rocha, Vanina Mollo et François Daniellou

\section{NOTE DE L'ÉDITEUR}

Article soumis le15/03/2017, accepté le 28/07/2017

Cette recherche n'aurait pas été possible sans le fort engagement de plusieurs acteurs de l'entreprise. Nous remercions vivement tous les travailleurs impliqués dans ce travail. Nous remercions également les relecteurs de cet article pour leurs remarques constructives.

\section{Introduction}

1 Malgré les importants progrès réalisés au cours des dernières décennies, l'évolution des systèmes sociotechniques appelle un développement toujours croissant des moyens mis en œuvre pour assurer la sécurité. De nouvelles règles, de nouveaux outils et de nouvelles technologies ont été créés pour accompagner cette évolution, ce qui a amené les salariés à connaître différents types de changements dans leur travail. D'une part, les managers opérationnels se retrouvent de plus en plus soumis à l'obligation de résultats managériaux, s'éloignant ainsi de la scène du terrain (Detchessahar \& Journé, 2007). D'autre part, les procédures de sécurité se multiplient dans le but de couvrir toutes les situations prévisibles, et peuvent mettre les salariés dans des situations où le respect des 
règles devient difficile, voire impossible. Les travailleurs sont donc amenés à violer certaines règles de sécurité pour garantir la performance du système et pour faire sortir l'entreprise d'une situation non anticipée (Amalberti, 2013).

Dans le même temps, les modèles et les outils actuels de prévention des accidents sont anciens et généralement focalisés sur les sanctions des «mauvais comportements » des opérateurs sur le terrain. Dans ce contexte, les travailleurs sont conduits à ne plus remonter les situations critiques ou celles qui s'éloignent du prescrit (Daniellou, Simard, \& Boissières, 2010). Ce modèle se révèle inadapté face à la complexité des systèmes actuels.

Pour avancer dans le domaine de la sécurité, différentes recherches mettent l'accent sur l'importance de revitaliser le collectif de travail et de mettre en discussion le travail réel au sein des organisations pour sortir du silence organisationnel, pour être au plus près de la réalité du travail et pour produire des règles adaptées (Detchessahar, 2001; Rocha, Mollo, \& Daniellou, 2015 ; Van Belleghem \& Forciolli Conti, 2015).

Dans ce sens, une entreprise de distribution d'énergie électrique a souhaité faire évoluer sa politique de gestion de la sécurité caractérisée par un système de contrôle lourd (de nombreuses règles et procédures, des sanctions et pénalités rigides sur le non-respect des règles, etc.) et, malgré tout, des anomalies de terrain plus ou moins visibles par l'organisation. La recherche-action décrite dans cet article avait pour objectif de développer, au sein de cette entreprise, des espaces dans lesquels les situations de terrain plus ou moins délicates pourraient être débattues collectivement entre les salariés, mais également entre les salariés et les managers, afin d'agir sur l'organisation. L'objectif était également de "structurer » ce débat, c'est-à-dire de l'organiser de manière qu'il fasse écho dans les différentes couches managériales de l'entreprise.

Après avoir présenté les principales notions sur lesquelles s'est appuyée cette recherche, le dispositif de mise en débat expérimenté à tous les niveaux de l'organisation sera présenté. Nous développerons ensuite les contributions de ce dispositif, à la fois au niveau individuel et organisationnel, ainsi que les conditions à respecter pour qu'il permette le développement d'un environnement capacitant, c'est-à-dire, celui capable de favoriser le développement des compétences et le pouvoir d'agir des individus pourqu'ils aient plus possibilités de contrôle sur leur tâche ${ }^{1}$.

\section{Les espaces de discussion et la subsidiarité pour faire face au silence organisationnel}

\subsection{Le silence organisationnel : des conséquences néfastes pour les individus et l'organisation}

6 La littérature scientifique autour du terme « silence organisationnel » n'est pas ancienne ${ }^{2}$. Cette notion qualifie les entreprises dans lesquelles le choix dominant fait par les employés est de garder pour eux leurs opinions et préoccupations par rapport aux problèmes organisationnels (Morrison \& Milliken, 2000, p. 707). Pinder et Harlos (2001), quant à eux, définissent le silence organisationnel comme un choix intentionnel de ne pas communiquer des informations critiques sur l'organisation à d'autres membres capables de changer ou redresser ces aspects. Plus récemment, et dans la même optique, le Groupe de travail de l'Icsi «Culture de sécurité» (2017, p. 81) affirme que «le silence 
organisationnel désigne une situation où des informations importantes pour la sécurité sont disponibles au niveau du terrain, mais ne remontent pas ».

7 Ces définitions montrent que le silence de l'employé est le fruit d'une décision consciente et délibérée, et que les informations cachées sont souvent critiques (Van Dyne, Ang, \& Botero, 2003). Or, la motivation qui amène les travailleurs à rompre ou pas ce silence est directement liée aux conditions que l'organisation et les managers offrent pour développer leur parole (Dejours, 1998). Morrison et Milliken (2000) affirment que le silence est dû à l'absence d'humilité managériale héritée de parcours scolaires prestigieux, ce qui nie la possibilité d'une intelligence au plus près du terrain. Selon ces auteurs, dans les entreprises où règne le silence, on retrouve les caractéristiques suivantes : les managers considèrent les employés comme peu fiables, les retours négatifs venant du bas sont délégitimés et la critique est une menace et une remise en cause de leurs propres compétences. Dit autrement, l'accord est donc un signe de bonne santé de l'organisation alors que le désaccord doit être empêché.

Du point de vue des opérateurs, deux raisons découragent toute forme d'expression: la peur de répercussions négatives auxquelles peut amener le fait de soulever certaines questions critiques internes à l'organisation, et le sentiment que la remontée d'information n'est pas suivie d'actions aux niveaux supérieurs (Morrison \& Milliken, 2000 ; Groupe de travail de l'Icsi « Culture de sécurité », 2017). L'individu, en craignant la façon dont les managers ou les pairs recevront le message, et en étant convaincu que les cadres sont peu disposés à écouter ou à prendre des mesures correctives, ne divulgue pas les informations puisque de toute façon il pense qu'il n'y aura aucun effet (Dejours, 1998).

9 Du point de vue des managers, le silence peut s'expliquer par des stratégies de défenses qui consistent à interrompre inconsciemment la remontée d'informations du terrain lorsque ces dernières sont en contradiction avec les injonctions provenant de la direction. Il peut également s'expliquer par une faible connaissance technique du terrain qui ne permet pas toujours de mesurer l'enjeu des alertes qui lui parviennent ; par l'idéologie du «bon professionnel » qui conduit à penser qu'un bon professionnel n'a pas de problème ou, s'il en a, doit être en mesure de le régler de façon autonome; ou par l'illusion de maitrise de l'organisation, ou la croyance selon laquelle les procédures couvrent tous les cas et si quelqu'un s'est trouvé dans une situation dangereuse, c'est forcément parce qu'il n'a pas respecté une procédure (Groupe de travail de l'Icsi « Culture de sécurité », 2017).

Le silence organisationnel peut avoir des conséquences néfastes sur le fonctionnement d'une organisation dans son ensemble mais aussi sur le bien-être de ses membres (Tangirala \& Ramanujan, 2008). Pour les individus, ne pas développer les conditions pour les retours d'expérience peut provoquer un sentiment de sous-valorisation et les amener à une démotivation, une insatisfaction et un désengagement du travail (Vakola \& Bouradas, 2005). Une souffrance significative qui peut se manifester à travers la hausse du stress au travail, jusqu'à conduire à des troubles psychologiques plus graves comme la dépression (Tangirala \& Ramanujan, 2008). Le plaisir et la motivation pour la profession tendent à perdre de leur intensité lorsque l'individu ne sent pas qu'il peut participer activement au bon fonctionnement de son entreprise (Conlon, Meyer, \& Nowakowski, 2005). Du point de vue de l'entreprise, un certain nombre d'analyses d'accidents mettent en avant les difficultés, les déficits et les lacunes de communication entre les salariés comme étant des causes de survenue de plusieurs catastrophes (Llory, 1996). Le fait de se taire renforce les croyances managériales selon lesquelles les individus sont peu fiables et désintéressés de la bonne marche de l'entreprise. Ainsi, la prise de décision devient le 
résultat d'une seule vision, celle des cadres, qui ont souvent peu d'informations sur la réalité empirique du terrain.

\subsection{Les espaces de discussion comme condition pour développer des organisations capacitantes}

11 Rompre le cercle vicieux du silence organisationnel, c'est avant tout se donner les moyens de profiter de la richesse de la diversité, en créant des systèmes qui donnent la parole aux employés (Daniellou \& Garrigou, 1993 ; Falzon \& Mollo, 2009). Des disciplines comme les sciences de gestion, la psychologie du travail et l'ergonomie développent depuis presque 20 ans des modèles autour des espaces de discussion. Selon ces théories, accompagner l'évolution du monde du travail suppose de réinstaurer des espaces de confrontation et de médiation via la discussion sur le travail entre les différents acteurs de l'organisation, pour gérer les conflits du point de vue de la santé et sécurité des travailleurs, et de la performance de l'entreprise. Il s'agit d'une institutionnalisation des moments de discussion qui, d'une entreprise à une autre, peut prendre différentes formes et s'incarner dans différents dispositifs (Detchessahar, 2011).

Dans cette optique, Detchessahar (2001) développe la «théorie des espaces de discussion » en préconisant la discussion autour du travail, dans le plan d'action, de façon régulière et protégée, animée par un cadre du métier n'appartenant pas à la ligne hiérarchique. Il s'agit d'une mise en débat bien définie qui doit se développer en des lieux assez structurés par des outils, des architectures ou encore des systèmes d'information qui visent à soutenir, faciliter ou informer la discussion de manière à la rendre la plus productive possible.

Pour traiter les questions de sécurité, santé et performance, le cœur de la discussion dans ces espaces doit tourner autour du travail réel et des adaptations mises en œuvre par les travailleurs pour faire face aux situations qui n'ont pas été anticipées. Il s'agit d'un "medium à travers lequel se réalise l'ensemble des arrangements, compromis et bricolages que supposent l'incomplétude de la prescription et le caractère irréductiblement erratique de l'activité concrète » (Detchessahar, 2013, p. 59).

Or, la discussion sur le travail peut amener au développement des organisations capacitantes, ou celles qui offrent les moyens aux opérateurs d'apprendre par leur propre activité (Arnoud, 2013 ; Barcellini, 2015 ; Falzon, 2013). Même si cette notion n'est pas tout à fait figée (Barcellini, 2015), on observe qu'elle est très proche de celle "d'environnement capacitant ", c'est-à-dire un environnement qui préserve les capacités futures d'agir de l'individu, permettant aux personnes et aux collectifs de mettre en œuvre leurs capacités de façon efficace et de développer de nouveaux savoir-faire et de nouvelles connaissances (Falzon, 2013). Selon Arnoud (2013), la mise en place d'un environnement capacitant est un facteur de conversion des ressources en capabilités (le fait d'être en mesure de faire quelque chose). La pérennisation de cette conversion n'est possible qu'à travers une " organisation capacitante ».

Barcellini (2015) soutient qu'une organisation capacitante favorise à la fois le développement d'une activité permettant aux opérateurs d'apprendre, et le développement de moyens qui permettent à une organisation de se reconfigurer et de se renouveler de manière continue. Encore selon l'auteure, «le développement de cette organisation doit être soutenu par l'existence d'espaces de discussion, d'objets 
intermédiaires soutenant le travail collectif au sein de ces espaces, mais également la possibilité de mobilisation d'acteurs ad hoc » (p. 50).

Dans cette optique, Arnould (2013) souligne qu'une organisation capacitante est une organisation qui donne la possibilité à chacun de construire sa propre activité, et qui «favorise la confrontation des points de vue en encourageant les débats à tous les niveaux de l'organisation » (p. 143).

\subsection{Un outil pour développer la subsidiarité}

17 L'idée d'un espace où les gens puissent s'exprimer existe dans le Code du travail français depuis 1981 (articles L2281-1 et suivants) à travers la loi du «droit d'expression » qui souligne le droit individuel de s'exprimer collectivement au sein d'un groupe homogène de travail. Toutefois, ce droit à la parole officielle des collectifs de travail s'avérait rapidement inefficace et plusieurs groupes d'expression en ont fait des expériences malheureuses (Linhart, 1991). Cela peut s'expliquer par deux raisons. D'abord, parce que les différents collectifs avaient du mal à se mettre d'accord sur les changements souhaités, et par conséquent, l'objet principal de la discussion n'était presque jamais le travail lui-même (Linhart, 2004). Ensuite, parce que lorsque des situations du terrain sont remontées et discutées, cela peut mettre en difficulté les managers de proximité qui n'auront pas toutes les réponses et toutes les ressources pour régler les problématiques survenues (Daniellou, 2013). C'est surtout de cette deuxième raison que traiteront les prochains paragraphes.

Les managers ont un rôle très important dans la mise en place d'espaces de discussion sur le travail. Ce sont eux qui vont gérer l'articulation entre le contenu des situations travaillées localement et les informations descendant des échelons supérieurs. Ces éléments nous amènent à réfléchir à deux questions essentielles. D'une part, le manager qui fait vivre le débat à son niveau peut faire émerger des problèmes pour lesquels il ne dispose pas de ressources de traitement suffisantes. D'autre part, le manager ne peut faire discuter du travail qu'à condition qu'il dispose lui-même d'un espace au niveau supérieur lui permettant de rendre compte du contenu de la discussion locale (Detchessahar, 2013).

19 Ces questions, qui traversent toute la ligne managériale, peuvent être mises en lien avec le principe de la subsidiarité, qui renvoie aux notions de "pouvoir » et «d'autonomie » des groupes sociaux. Cette notion, issue des sciences politiques, définit les principes de distribution du pouvoir dans une communauté et son principal intérêt est de penser à la fois la liberté et l'autorité des individus (Millon-Delsol, 1993). Dans le monde des entreprises, la subsidiarité se traduit par le fait qu'un échelon managérial supérieur ne doit pas exercer des fonctions qui peuvent être efficacement réalisées par un échelon inférieur. Pradines (2004) développe trois autres principes de cette notion dans les organisations: le principe de compétence, le principe de secours et le principe de suppléance. Dans le principe de compétence, l'échelon supérieur s'interdit toute tâche qui peut être accomplie par l'échelon inférieur. Les décisions doivent donc être prises au plus bas niveau possible. Par le principe de secours, l'échelon supérieur a le devoir de s'acquitter des tâches que l'échelon inférieur ne peut pas réaliser. Quand un niveau n'a donc pas les ressources pour traiter une question, le niveau supérieur doit la prendre en charge. Enfin, dans le principe de suppléance, l'échelon inférieur s'interdit de se décharger de certaines tâches qui lui reviennent en propre. Cela veut dire que lorsqu'un niveau a les ressources pour traiter une question, il ne peut pas se dérober. 
Le principe de la subsidiarité renvoie ainsi à la « recherche permanente du niveau le plus pertinent pour l'action » (Petit, Dugué, \& Daniellou, 2011, p. 404), à partir de l'articulation et de l'ajustement des différents collectifs de l'organisation. Il permet de développer le collectif par la réflexion autour des notions de pouvoir et d'autonomie, de façon à permettre à chaque niveau de garder sa capacité d'agir.

\section{Méthodologie}

L'entreprise étudiée dans cette recherche, située dans les Pays de la Loire, possède 28 sites de distribution d'énergie électrique et totalise environ 600 agents. Le travail consiste à relever des compteurs, mettre en service ou hors service l'électricité, changer des disjoncteurs et des tableaux électriques, effectuer des dépannages et des branchements sur le réseau.

La démarche s'est structurée en deux grandes phases : une phase d'expérimentation des espaces de débat sur le travail (EDT) et une phase d'organisation de la subsidiarité et de généralisation de la démarche.

\subsection{Phase d'Expérimentation des EDT}

La phase d'expérimentation des EDT a fait suite à un diagnostic ergonomique au cours duquel un ensemble de facteurs qui conduisaient au silence organisationnel ont été décrits, expliqués et validés ${ }^{3}$. Elle a été conduite au sein d'un site composé de 6 techniciens et d'un manager de proximité.

Dans un premier temps, l'ergonome co-animait le débat en collaboration avec le manager de proximité en se basant sur des situations concrètes issues du diagnostic. Quatre réunions séparées par des intervalles réguliers (toutes les 2 semaines) ont ainsi été réalisées. Parallèlement au traitement de ces situations, le groupe a défini une méthode de mise en débat quotidienne des situations de terrain, en tenant compte des caractéristiques organisationnelles locales (réunions et outils managériaux existants) et des contraintes des travailleurs (temps disponible pour la discussion et moyens existants pour régler les situations discutées).

Dans un second temps, le dispositif conçu par le collectif a été expérimenté. Le débat était alors animé par le manager de proximité, l'ergonome étant observateur.

Au total, 8 EDT ont été enregistrés, retranscrits et analysés. Une analyse thématique a permis d'identifier trois variables principales décrites dans le tableau $1:$ la dynamique du débat, le type de situation débattue, et la participation des interlocuteurs. Cette analyse a été complétée par un traitement quantitatif à l'aide du logiciel Actogram Kronos (Kerguelen, 2003). Les graphes fournis par ce logiciel ont permis de représenter l'état des différentes variables pour chacune des séances, mais aussi l'évolution de ces variables au fur et à mesure de l'avancée des séances .

Tableau 1 : Variables d'analyse du contenu des EDT.

Table 1: Variables for analyzing the content of Work Debate Spaces

\begin{tabular}{|l|l|l|}
\hline Variables principales & Sous-variables & Définitions \\
\hline
\end{tabular}




\begin{tabular}{|c|c|c|}
\hline \multirow{2}{*}{ Dynamique du débat } & Analyse & $\begin{array}{l}\text { Analyse collective du risque, des origines et des } \\
\text { conséquences du dysfonctionnement. }\end{array}$ \\
\hline & Traitement & $\begin{array}{l}\text { Élaboration collective de solutions pour répondre } \\
\text { aux situations analysées. }\end{array}$ \\
\hline \multirow{3}{*}{ Sujets débattus } & $\begin{array}{l}\text { Situations de } \\
\text { terrain }\end{array}$ & $\begin{array}{l}\text { Le débat porte sur une situation particulière du } \\
\text { terrain. }\end{array}$ \\
\hline & $\begin{array}{l}\text { Situations } \\
\text { génériques }\end{array}$ & $\begin{array}{l}\text { Le débat porte sur un problème générique / une } \\
\text { famille de situations de terrain. }\end{array}$ \\
\hline & $\begin{array}{l}\text { Ingénierie des } \\
\text { EDT }\end{array}$ & Le débat porte sur l'organisation des EDT. \\
\hline \multirow{2}{*}{$\begin{array}{l}\text { Participation des } \\
\text { interlocuteurs }\end{array}$} & $\begin{array}{l}\text { Animateur } \mathrm{du} \\
\text { débat }\end{array}$ & $\begin{array}{l}\text { Celui qui parle est celui qui anime l'EDT: le } \\
\text { chercheur et/ou le manager de proximité. }\end{array}$ \\
\hline & Participants & $\begin{array}{l}\text { Celui qui parle est un membre de l'EDT : un } \\
\text { technicien }(\mathrm{TC} 1,2,3 \ldots) \text { ou le manager de proximité. }\end{array}$ \\
\hline
\end{tabular}

\subsection{Phase d'organisation de la subsidiarité et de généralisation de la démarche}

La phase d'organisation de la subsidiarité a consisté à déployer le traitement de situations de travail aux différents niveaux hiérarchiques de l'entreprise. En effet, le déploiement du dispositif local de mise en débat a permis de traiter un certain nombre de situations, mais les agents n'étaient pas toujours en mesure de régler une autre partie des situations discutées. Ceci nous a amenés à développer des dispositifs similaires au niveau des échelons supérieurs de l'entreprise, de façon à prendre en charge les situations qui ne pourraient pas être traitées localement. À chaque situation remontée au niveau supérieur, le management se demandait quelles seraient les ressources à fournir au niveau inférieur pour qu'il puisse traiter les situations similaires à venir. Ainsi, le but n'était pas d'avoir un nombre plus important de situations remontées, mais que le minimum remonté soit effectivement traité.

La phase de généralisation, quant à elle, a consisté à former et suivre les managers de l'entreprise sur l'approche développée. Deux types de formations ont été réalisées : une formation-action au Comité de Direction (Codir) local (10 managers, l'Expert prévention et le Directeur de l'unité) et une autre au Codir régional (16 directeurs d'unités). La première formation était composée de 2 journées successives complétées d'une troisième journée 9 mois après. L'objectif était de rendre les managers capables de faire vivre la démarche, de co-construire avec eux le dispositif systémique (qui comporte plus d'un niveau hiérarchique de l'entreprise) de mise en débat afin de le tester dans leur site, et de restituer les résultats et les difficultés rencontrées au moment de la $3^{\mathrm{e}}$ journée. La deuxième formation, quant à elle, était composée de 2 journées successives. Les principaux objectifs étaient de partager les concepts et principes majeurs de la prise en compte d'une démarche facteurs humains et organisationnels (FHO) pour consolider le 
sens de la démarche et des actions en cours. Pour les participants, c'était aussi un moyen de connaître quelques résultats de la recherche engagée afin de les faire progresser et de la diffuser auprès d'autres sites et d'autres métiers de l'entreprise.

\section{Résultats}

\subsection{Le dispositif de mise en débat sur le travail : contributions locales}

L'analyse du travail menée auprès des techniciens et du manager de proximité a mis en évidence une rupture du collectif et l'absence de dialogue sur le travail entre les techniciens et les niveaux hiérarchiques supérieurs. Les premières séances de mise en débat du travail confirment ces données. Les techniciens s'expriment sur le fait que le système actuel de remontée des situations dangereuses (SD), qui fixe des objectifs annuels (2 SD par an par technicien), ne fait pas sens pour eux puisque la plupart des agents le font juste pour remplir cet objectif. De plus, les techniciens ont toujours peur d'être sanctionnés en fonction de ce qui est remonté. Ils expliquent qu'ils remontent des situations faibles du point de vue des risques, et qu'ils gardent pour eux les "vraies " situations dangereuses dans lesquelles ils peuvent être mis en cause, principalement celles qui peuvent témoigner d'erreurs de leur part. Les propos du manager de proximité légitiment cette question dans la mesure où la procédure d'évaluation annuelle peut constituer une forme de sanction. Il déclare en effet que si dans l'entretien d'évaluation annuel un technicien remonte une erreur, il ne sera pas inclus dans le groupe qui bénéficiera d'avancements.

Au cours des séances, le groupe a exprimé le besoin de développer un système de partage et de traitement des anomalies de terrain. Nous avons ainsi co-construit un dispositif local avec tous les agents de ce site. Ce dispositif a pris un format « cyclique » comprenant 4 étapes représentées dans la figure 1 :

- Les techniciens prennent des photos des événements significatifs ${ }^{5}$ (ES) du terrain. Ces derniers concernent soit des situations qu'ils considèrent anormales (anomalies), soit des adaptations ou stratégies mises en place ;

- Les photos prises sont transmises au manager de proximité lors du débriefing quotidien ;

- Le manager de proximité peut éventuellement faire un tri des situations considérées les plus pertinentes pour les mettre en discussion collectivement ;

- Le manager de proximité anime un débat collectif dans les « réunions de groupe » (qui ont lieu une fois par semaine) à partir des situations remontées et triées. Il trace le sujet discuté et les actions décidées dans un tableau co-construit avec le groupe. 
Figure 1 : Dispositif local de débat conçu avec les participants du site.

Figure 1: Local discussion system designed with the participants of the site

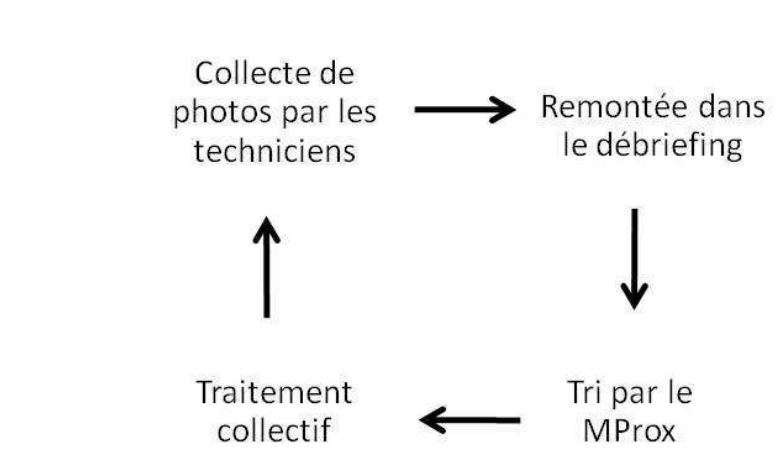

Niveau site

Ce dispositif a apporté plusieurs contributions pour le groupe de l'unité concernée, qui sont détaillées ci-dessous : l'amélioration de la déclaration et du traitement de situations à risque, le développement du collectif de travail et une dynamique d'apprentissage entre les participants.

\subsubsection{Amélioration de la déclaration et du traitement de situations à risque}

L'analyse des débats montre un impact direct de la mise en place des EDT sur le nombre de déclarations des situations à risque, le temps et la qualité de leur traitement sur le site. $\mathrm{Si}$, avant la mise en œuvre de notre approche, chaque technicien remontait 1 à 2 situations par an (conformément à l'objectif prescrit par l'entreprise), maintenant chaque technicien remonte 2 à 3 situations par mois. L'analyse du contenu des EDT montre que dans les premières séances, les situations remontées font l'objet d'une analyse de leurs causes et conséquences, mais au détriment de leur traitement. Cela peut s'expliquer par le fait que les agents disposent désormais d'un espace formel pour discuter des situations de terrain qu'ils n'avaient pas auparavant. Au fur et à mesure des séances, on constate un équilibre de plus en plus fort entre l'« analyse » et le «traitement » des situations. Si le nombre de situations remontées et leur temps de traitement augmentent, la qualité de ce traitement évolue également. Auparavant, les techniciens remontaient les situations dans le système informatique de l'entreprise, et le traitement de ces situations était assuré par l'expert prévention. Avec les EDT, la situation est analysée et traitée par le groupe, sans forcément être saisie dans le système informatique. Cela signifie que lorsque le groupe a les ressources nécessaires localement pour traiter les anomalies remontées, il les traite au lieu de renvoyer le traitement à l'expert prévention. Dit autrement, si la déclaration et la gestion d'une situation étaient deux processus distincts entre lesquels il n'existait pas de feedback, désormais le collectif la discute, anticipe les risques possibles et définit des solutions.

\subsubsection{Développement du collectif de travail}

33 L'EDT a contribué également au développement du collectif de travail local. Premièrement, la confrontation régulière des points de vue permet aux agents de faire converger leurs appréciations du risque. Le fait de pouvoir discuter du risque et de rechercher des solutions collectives amène les agents à créer des règles communes et partagées. C'est le cas par exemple pour une situation discutée où le client a détaché son 
tableau électrique du mur et l'a mis sur une chaise, en laissant les fils sous-tension exposés. Les agents se sont accordés pour disposer en permanence de ruban adhésif et d'autocollants dans les véhicules afin de traiter des situations similaires dans le futur. Deuxièmement, le débat a permis le développement du soutien individuel et collectif. Le partage d'une situation quotidienne vécue est rassurant non seulement pour celui qui la remonte, qui peut observer qu'il n'est pas le seul à la vivre, mais aussi pour les collègues qui sont susceptibles de rencontrer une situation similaire à l'avenir, et qui seront mieux préparés pour y faire face. Dans le même temps, au travers du débat, le manager de proximité accède à la réalité du travail des techniciens, à leurs difficultés, à leurs appréciations du risque, aux adaptations mises en place et aux modalités de traitement des situations discutées. Les techniciens peuvent se confier à lui puisqu'il est dans une logique d'essayer de comprendre et de trouver des solutions avec eux. Troisièmement, les EDT permettent de développer la confiance au sein du groupe. Au fil des séances, les agents libèrent progressivement leur parole et partagent de plus en plus de situations vécues individuellement, gagnant une confiance mutuelle. Ce résultat est d'autant plus marquant pour certains techniciens qui n'intervenaient pas beaucoup au début des réunions, mais qui, dans la phase d'expérimentation du dispositif, prennent souvent la parole. On observe également une tendance progressive à l'échange entre techniciens qui, lors des premières réunions, intervenaient le plus souvent en réponse à l'animateur de la séance. Désormais, ils se questionnent mutuellement, interviennent sur les explications du collègue et prennent beaucoup plus d'initiatives pour changer ou introduire les sujets de la discussion.

\subsubsection{Développement d'une dynamique d'apprentissage}

Le partage des situations vécues et leur gestion a permis aux agents à la fois d'apprendre au groupe et d'apprendre par le groupe, comme en témoignent quelques techniciens: "les choses qu'on discute ici, je n'ai pas vu dans ma formation»; "maintenant je comprends»; «je ne savais pas». L'analyse des débats montre qu'un premier temps consiste à élaborer des hypothèses sur ce qui a pu conduire à une telle situation, et sur les conséquences éventuelles. Puis les agents échangent sur le risque de la situation. L'appréciation du risque diffère selon les acteurs et conduit chacun à argumenter son propre point de vue. Cette confrontation des appréciations n'aboutit pas nécessairement à un consensus, mais permet un partage de logiques qui peuvent être divergentes. Le fait de connaître d'autres points de vue peut conduire les opérateurs à réfléchir de façon plus approfondie ou à considérer de nouveaux éléments pour traiter certaines situations. Or, en parlant plus et en donnant plus souvent des exemples de situations passées ou des situations futures probables, les techniciens créent les moyens d'apprendre à leurs collègues et de leurs collègues, comme le montre l'exemple ci-dessous, lors que le «technicien 2 » exprime sa compréhension da la situation expliquée par le «technicien 1 ».

Exemple d'une dynamique d'apprentissage d'après la situation débattue

«Donc, voilà la $1^{\text {re }}$ SD que vous avez remontée. Je vous laisse lire (...) ». Chercheur.

«C'est pas réglementaire déjà, il n'y a pas de CC (boîte court-circuit) ». Technicien

1.

«Concrètement, ils ont fait quoi ? » Technicien 2.

«En fait en dessous il n'y a pas de boîte CC, il est pris directement sur le branchement ». Technicien 1.

«Donc, s'il y a un incident sur le branchement on ne peut pas couper. Au moins 
directement ». Expert Prévention.

«Et après ça tourne dans le coffret là ? Ce petit... ». Technicien 2.

«Non. Ça n'a rien à voir. Le coffret c'est de la maison à gauche et le CBI c'est de la maison de droite ». Technicien 1.

« D'accord. J'ai compris ». Technicien 2.

«Et c'est un CBI intégré ? ». Expert Prévention.

« Non, non, il n'y avait pas en plus. C'est le $1^{\mathrm{er}}$ modèle ». Manager de Proximité.

« Non. Il n'y avait pas de CC ». Technicien 1.

« Donc, à l'intérieur ils ont mis un tableau sans CC?». Technicien 2.

«Non, non, même pas. Le $1^{\text {er }}$ modèle à l'intérieur tu mets un tableau 25-50 sur lequel il avait un CC. Dans certains centres ils utilisaient déjà le fusible grenade (fusible particulier pour certains tableaux de comptage), mais ici n'avait pas les conditions pour la mise en place ». MProxSénior.

«Ah oui, d'accord ». Expert Prévention.

«Et donc l'agent ou le technicien l'ouvre?». Technicien 2.

«Il faudra enlever le compteur, quoi ». Technicien 1.

«Ah ouais! Il fallait enlever les patates à l'intérieur, ok, je comprends ». Technicien 2.

\subsection{Le dispositif systémique de mise en débat sur le travail : contributions globales} situations débattues, comme des remplacements de portes de coffrets cassées, des demandes de réparation au service d'exploitation du réseau ou la collaboration avec les prestataires sur certaines interventions techniques qui posaient problèmes. Au début de chaque séance, le manager de proximité fait un retour au groupe sur les actions décidées lors de la réunion précédente, celles qui ont été mises en place et celles qui sont en suspens. Les techniciens manifestent que ce point d'information est essentiel pour la poursuite et la pérennisation de cette démarche puisqu'ils ont « besoin de savoir ce qui se passe suite aux décisions prises ensemble ».

Toutefois, les agents n'ont pas toujours les moyens nécessaires pour gérer toutes les situations remontées. Selon le manager de proximité, les principales difficultés du dispositif mis en place concernent «la difficulté de trouver le bon interlocuteur pour résoudre un problème qu'on ne peut pas gérer sur place » et « le fait d'avoir un retour des services concernés lorsqu'on trouve un interlocuteur ». Le groupe exprime ainsi le besoin de remonter certaines situations à un autre niveau afin d'être soutenu dans son analyse et sa gestion. Ce besoin constitue en effet une condition principale pour que la démarche soit pérennisée au sein de l'entreprise. Si aucun interlocuteur d'un niveau hiérarchique supérieur n'est en mesure de récupérer les situations non gérées sur place et de donner suite à leur traitement, l'EDT risque très fortement de s'essouffler.

Dans cette optique, en accord avec la direction et l'expert prévention de l'entreprise, il a été décidé que les situations remontées seraient traitées au niveau du service prévention, qui assurerait un feedback des actions menées au niveau local (comme le manager de proximité dans le dispositif local de mise en débat). Ainsi, nous avons construit ensemble une deuxième boucle du dispositif local de mise en débat, représentée dans la figure 2. 
Figure 2 : Schéma montrant la remontée d'informations au niveau de la prévention. Figure 2: Diagram showing information-gathering at prevention level

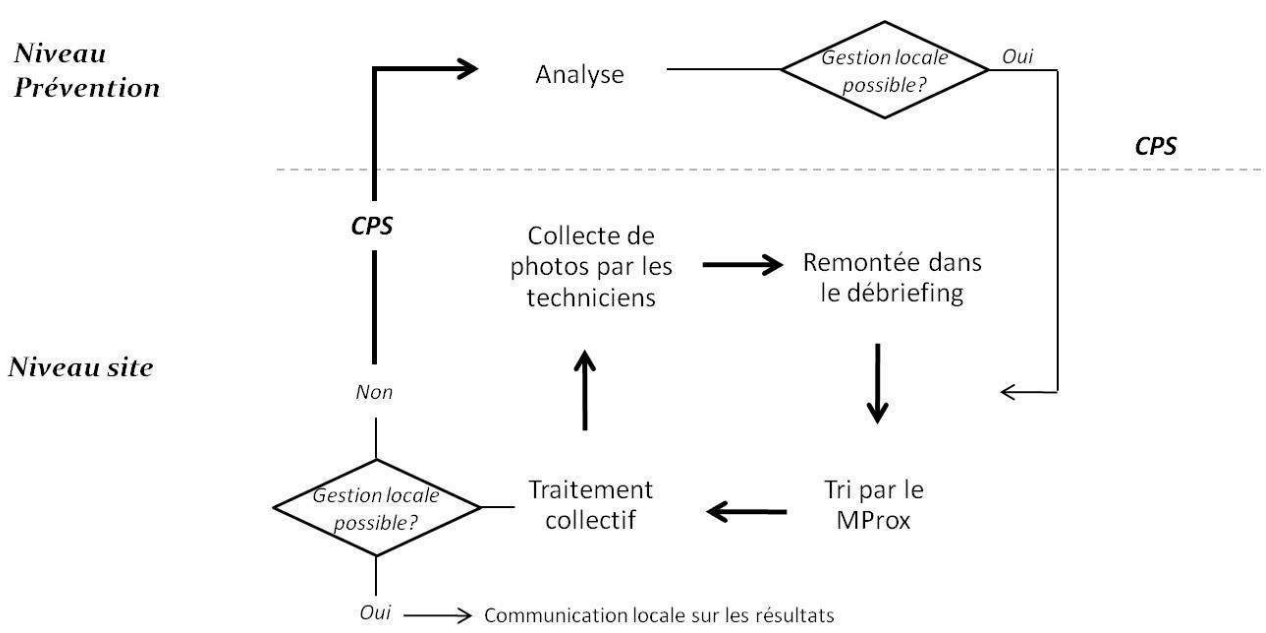

Tout comme pour le dispositif local, l'objectif était de profiter de la structure existante pour construire cette étape. Nous avons donc décidé de nous appuyer sur la réunion trimestrielle du Comité Prévention Sécurité (CPS), qui rassemble l'expert prévention et les responsables des sites dans le but de transmettre des informations sécurité (stratégiques et résultats). Un temps a été dégagé pour que le manager de proximité du site puisse remonter les situations non traitables localement à ce groupe. Cette réunion aurait également deux autres objectifs : elle serait le moment où l'expert prévention pourrait faire un retour sur les situations traitées précédemment; et elle serait l'occasion de généraliser les EDT dans la mesure où le débat en CPS est un moyen pour les managers de proximité des autres sites de s'approprier la démarche.

Or, si le niveau de la prévention contribue à régler certaines situations, il n'a pas non plus les moyens de régler tout ce qui est remonté. Pour soutenir le CPS, les réunions trimestrielles du Codir (auxquelles participe l'expert prévention) ont été choisies, en accord avec le directeur de l'entreprise, pour traiter les événements significatifs qui ne peuvent être traités ni localement, ni au niveau de la prévention. La figure 3 présente le dispositif systémique construit auprès des différents salariés de l'entreprise. 
Figure 3 : Schéma démontrant la remontée d'informations au niveau supérieur. Figure 3: Diagram showing information-gathering at higher level

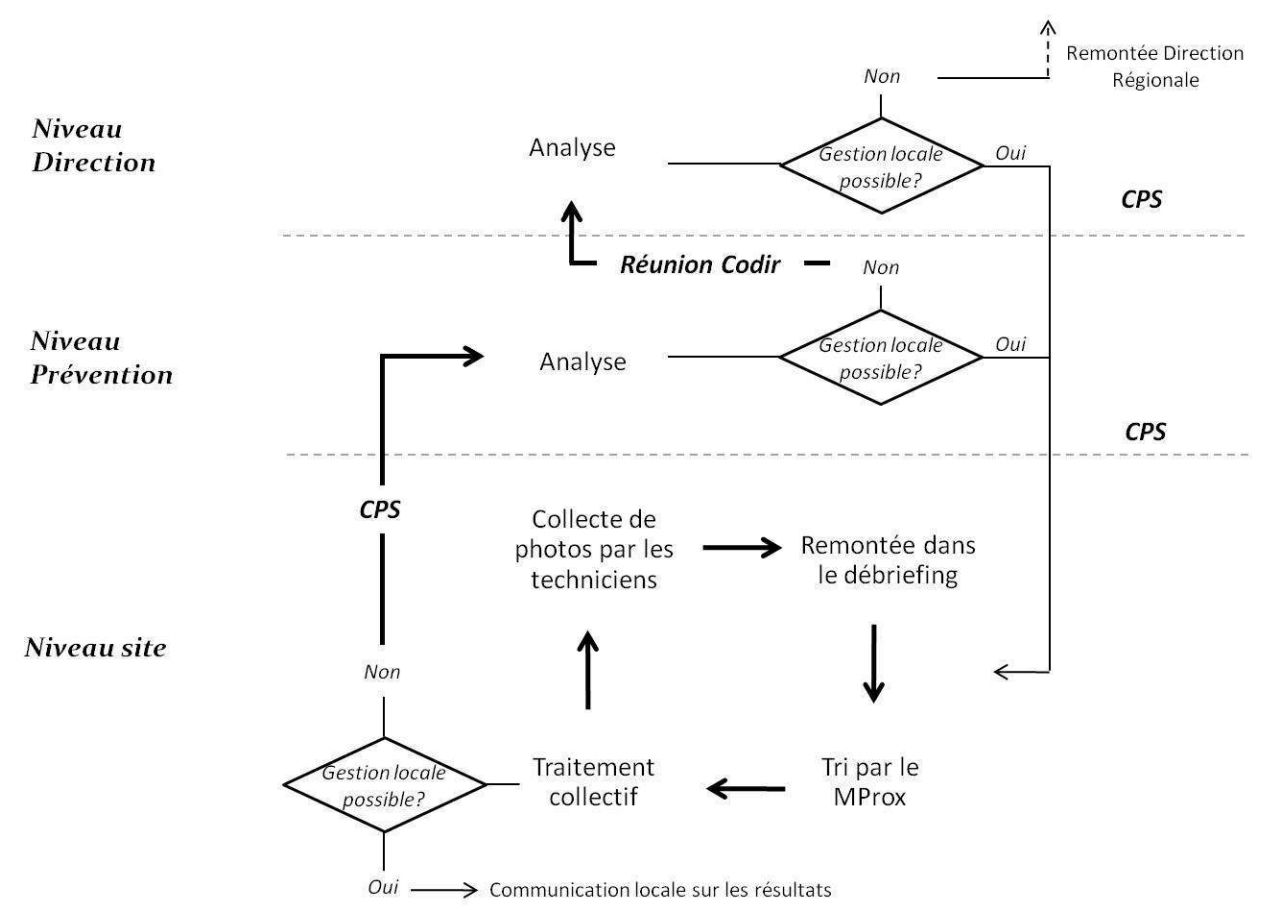

41 Lors de ces réunions, quelques minutes sont consacrées à la présentation par l'expert prévention des situations les plus pertinentes discutées dans la réunion du CPS. Ce processus a permis à l'entreprise de traiter ce qu'elle appelle des «situations-type », ou des anomalies rencontrées régulièrement par les techniciens sur le terrain, soit parce que le Codir n'était pas au courant, soit parce que la complexité de la solution fait que cette anomalie résistait sur le terrain.

Si le Codir a le pouvoir de régler un certain nombre de situations qui lui sont remontées par les sites, il n'a pas non plus les moyens de toutes les gérer. D'autres instances devaient donc entrer dans la boucle de traitement, comme le niveau régional ou même national. Dans ce cas, le Codir choisirait l'interlocuteur pertinent pour dialoguer sur l'événement significatif. L'exemple ci-dessous illustre une situation qui a été remontée du site jusqu'au niveau national, concernant une innovation de matériel ${ }^{6}$.

\section{Exemple d'un événement significatif géré au niveau national}

Description : une clé à cliquet tombe de la sacoche d'un technicien qui intervenait en haut d'un poteau. Le technicien remonte la situation auprès de son manager de proximité. L'EDT réalisé au niveau du site fait ressortir des informations importantes : la sacoche actuelle n'est pas assez profonde et trop souple, ce qui crée un risque important de chute d'outils (Figure 4, photo de gauche); par rapport à l'ancienne (Figure 4, photo de droite), la sacoche actuelle a perdu en rigidité ; ce problème a déjà été identifié par plusieurs techniciens mais n'était pas forcément partagé avec le groupe ; certains techniciens se refusent à utiliser la sacoche et préfèrent utiliser un seau à la place. 
Figure 4 : Sacoche actuelle (photo de gauche) et ancienne sacoche (photo de droite). Figure 4: Current bag (left photo) and old bag (pictured right)

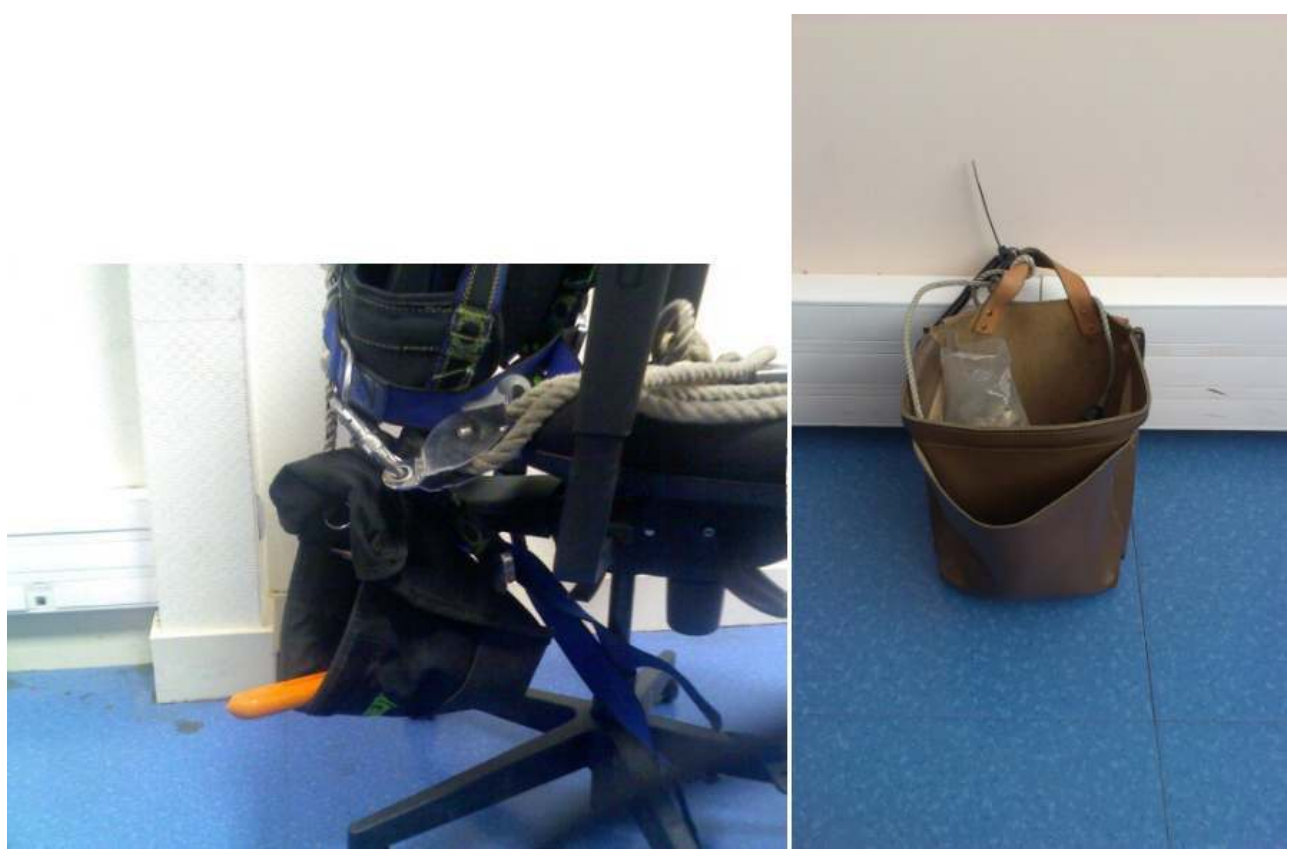

Certains techniciens avaient apporté des améliorations individuelles et artisanales sur la sacoche : un raccrochage entre deux sacoches par des fils de cuivre pour éviter qu'elles ne balancent (Figure 5, photo de gauche) ; une ouverture au niveau de la couture pour y glisser des tiges en plastique (des vieux pieds de coffret) afin de gagner en rigidité et réduire l'angle de balancier (Figure 5, photos du centre et de droite).

Figure 5 : Accrochage par des fils de cuivre ; adaptation avec des tiges en plastique. Figure 5: Re-hooking with copper wires; adaptation with plastic rods
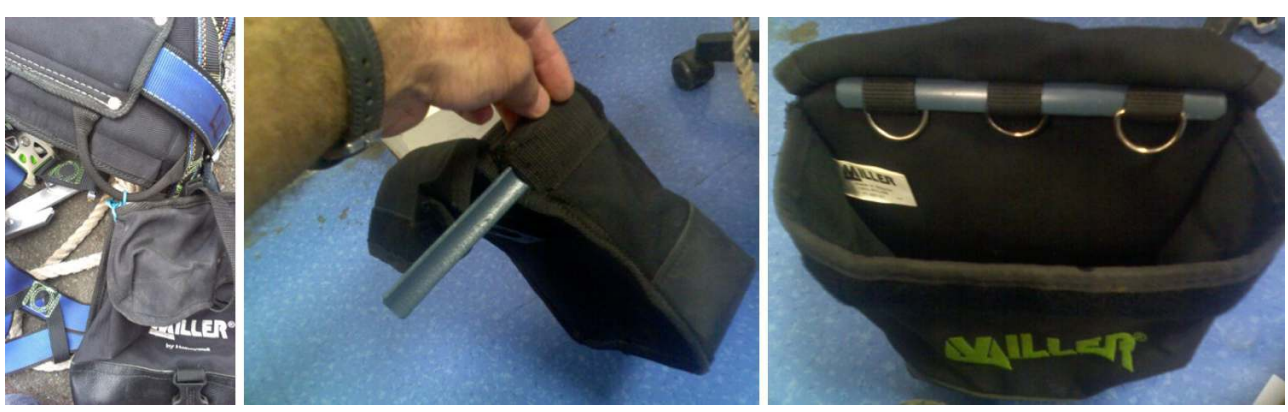

Actions : suite à ces éléments, le manager du site a décidé d'adapter toutes les sacoches avec des tiges en plastique pour améliorer leur rigidité : « nous avons étendu cette bonne pratique à toutes les sacoches en attendant d'avoir mieux». La situation a ensuite été remontée à la cellule prévention et au Codir. Les acteurs du Codir ont contacté le réseau " travaux en hauteur », en charge du matériel au niveau national. Suite à leur échange, le prescripteur national a mandaté un prestataire pour développer une sacoche plus adaptée à l'activité du groupe originaire de la demande. Un EDT a été organisé par ce prestataire et le responsable du site, afin de réunir les 
utilisateurs (les techniciens) et les concepteurs. Le débat a porté sur un prototype de sacoche plus sûr et moderne : plus légère, rigide, avec des rabats magnétiques. Le prototype réalisé a été validé par le groupe (Figure 6). Cette démarche a été valorisée dans l'entreprise comme une innovation, et a été diffusée auprès d'autres sites de l'entreprise en France.

Figure 6 : Sacoche produite entre les agents du site et l'entreprise prestataire. Figure 6: Bag produced by the agents of the site and the contractor

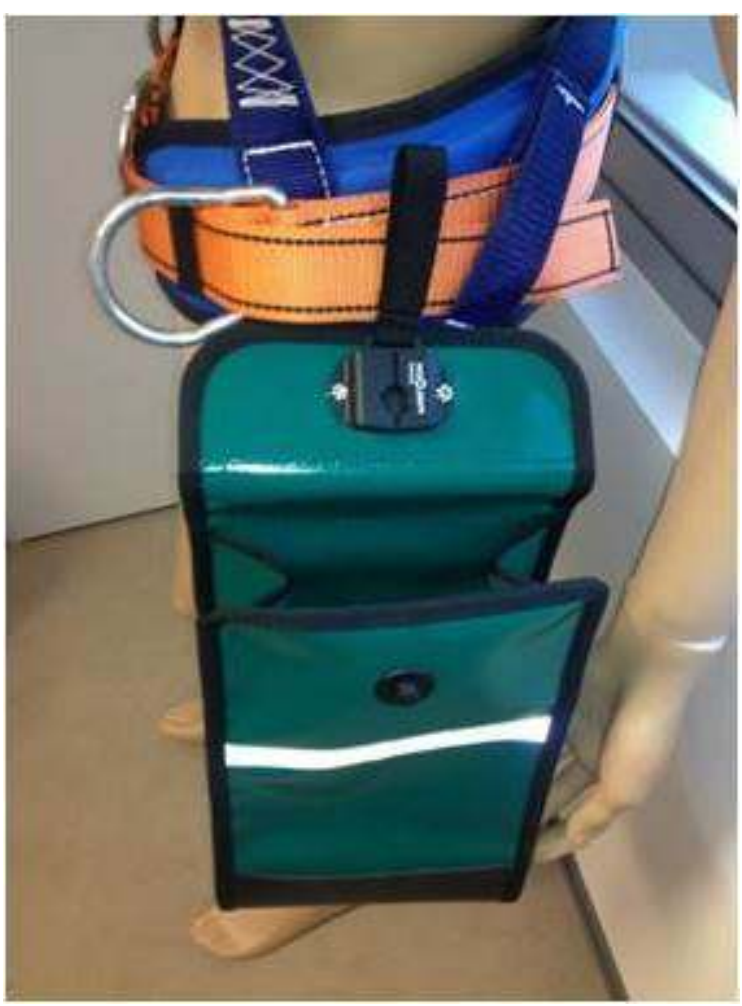

De même que le dispositif local, le dispositif systémique de mise en débat sur le travail a apporté quelques contributions, dans ce cas surtout pour l'organisation locale et globale. Ces contributions concernent principalement le développement de l'innovation et la performance, la reconnexion du management avec la réalité du travail et la convergence entre les règles, le discours et les pratiques managériales.

\subsubsection{Contributions à l'innovation et à la performance}

Le débat structuré sur le travail a contribué à l'innovation de l'entreprise et à améliorer les indicateurs d'innovation de l'unité travaillée. L'exemple discuté ci-dessus en est un exemple. D'une part, parce que l'on évite la multiplication des dépenses et l'aggravation des dégâts : l'ancienne sacoche ne protégeait pas assez les outils qui risquaient de tomber et de blesser les techniciens ou les clients, de se casser ou de casser les biens des clients (toit de la maison, voiture, portail, etc.), multipliant les coûts potentiels (dépenses de santé en cas de blessure des techniciens ou des tiers, achats de nouveaux matériels ou dépenses liées à la remise en état des dommages clients). D'autre part, l'amélioration apportée grâce au débat collectif permet d'éviter des accidents potentiellement très graves. La chute des outils de la sacoche peut endommager les objets du client mais peut aussi blesser très gravement le client lui-même. En évitant ces types d'accidents, on 
améliore la performance de l'entreprise dans le sens où l'on va éviter des dépenses économiques et améliorer l'image de l'entreprise vis-à-vis des clients. Cela va dans le sens de Detchessahar (2013) lorsqu'il affirme que la distribution et la connexion des espaces de discussion tout au long de la ligne hiérarchique sont un moyen puissant de ré-internaliser l'innovation et surtout d'offrir la possibilité de rétablir le travail comme origine possible de l'innovation.

\subsubsection{Reconnexion du management avec la réalité du travail}

L'approche développée a pu créer des conditions pour reconnecter le management de l'entreprise avec la réalité du travail. D'abord, le manager de proximité a toujours eu un rôle très important, non seulement comme garant de l'EDT, en l'intégrant systématiquement dans l'ordre du jour des réunions du groupe, mais aussi comme animateur de cet espace. Il porte attention à ce que chacun participe aux discussions et il interpelle si besoin les techniciens par des questions du type « tu peux nous en raconter un peu plus sur cette situation?» ou "comment vous réagiriez par rapport à cette situation?». Ce faisant, il accède à la réalité du travail des techniciens, aux différentes appréciations du risque, aux adaptations mises en place et aux modalités de traitement des situations discutées. Ensuite, lorsqu'une situation ne peut être réglée localement ou lorsque le manager de proximité considère pertinent de la diffuser à d'autres niveaux de l'entreprise, la situation peut être remontée jusqu'au niveau du Codir (voire plus). Une partie des réunions managériales est donc dégagée pour discuter de ces situations. $\mathrm{Ce}$ faisant, différents niveaux du management - comme le manager de proximité, les managers intermédiaires, les adjoints au directeur et les directeurs eux-mêmes discutent des cas concrets remontés du terrain, en les rapprochant du travail et des problèmes réels vécus par leurs équipes. Autrement dit, l'EDT a permis de rapprocher le management de l'entreprise à une réalité qui n'était pas bien claire auparavant.

\subsubsection{Convergence entre les règles, le discours et les pratiques managériales}

L'évolution du discours était le premier point de changement observé chez les managers. Lors du travail sur la demande de recherche, le discours de la direction était très axé sur le respect des règles et sur les comportements des agents. Dans la proposition de mission de cette recherche par exemple, il était attendu « d'agir durablement sur les mentalités et les comportements individuels et collectifs ». On constate une évolution progressive du discours tout au long de la recherche, comme le montrent les verbalisations des managers : « la démarche FHO cherche à traiter les soucis de l'organisation en prenant en compte les représentations des travailleurs opérationnels. C'est une approche complètement différente de la nôtre et qu'on souhaite bien s'approprier et mettre en place chez nous "; "à travers cette démarche, on cherche à changer notre posture managériale dans le sens de créer des conditions permettant aux travailleurs de nous remonter des situations de terrain dans un climat de confiance». Ensuite, des changements ont pu être observés au niveau des pratiques des managers de proximité d'une part, qui se sont rapprochés des techniciens afin d'échanger sur le travail réel, et des managers du Codir d'autre part, qui ont agi sur l'organisation pour pérenniser les pratiques de débat autour du travail réel. Si au début de la recherche, le manager de proximité parlait autant que les techniciens et avait un rôle plus discret, dans la phase d'organisation de la subsidiarité il joue le rôle de garant et d'animateur du débat. Les 
managers du Codir, à leur tour, ont dû s'organiser pour aider dans la réflexion sur le risque et la gestion des situations locales. Enfin, les procédures managériales ont également évolué. Des documents comme le «Plan d'action Prévention SantéSécurité 2013-2015» marquent maintenant l'importance de "déployer l'approche Facteurs Humains et Organisationnels comme un levier majeur de la culture PSS (Prévention Santé-Sécurité) ». De même, dans le document "Orientations Santé-Sécurité pluriannuelles 2013-2015 », 4 des 6 fondamentaux sont directement influencés par la recherche, à savoir : «garantir une présence managériale au plus proche des situations de travail», "valoriser la détection et la déclaration des erreurs et des situations dangereuses", "garantir un traitement exemplaire des situations dangereuses détectées » et « dialogue à tous les niveaux » de l'entreprise.

\section{Les conditions du débat structuré sur le travail}

\subsection{Conditions relatives au pilotage de la démarche}

Les conditions relatives au pilotage de la démarche concernent l'engagement du Codir à partir de la conscience des ses faiblesses, l'identification d'un référent FHO et des acteurs porteurs de la démarche, ainsi que la qualité du couplage Référent-Codir.

\subsubsection{Engagement du Codir à partir de la conscience de ses ressources et faiblesses}

L'engagement du Codir depuis le début de la démarche est évidemment fondamental. C'est lui qui octroiera les ressources et les moyens pour que les sites soient capables de mettre en place les espaces de débat, c'est lui qui travaillera pour " dés-empêcher » les managers de réguler le travail, et c'est lui qui décidera du degré d'autonomie de chaque niveau hiérarchique pour traiter les questions issues du terrain. Ceci étant, si l'engagement du Codir implique automatiquement son souhait, le souhait du Codir ne signifie pas nécessairement qu'il s'engage. Pour cela, il faut qu'il ait envie de changer sa politique de sécurité, notamment en prenant conscience de ses ressources mais aussi de ses faiblesses. Cela suppose d'être en mesure d'entendre d'autres points de vue qui peuvent remettre en cause certains actes du management autour de la sécurité. En effet, si l'espace de débat met en évidence l'activité réelle, il permet également de rendre visibles les vulnérabilités de l'entreprise et de mettre en lumière leurs origines profondes. De ce point de vue, l'entreprise peut refuser de (re)connaître ses vulnérabilités et inhiber cette pratique. La hiérarchie doit donc accepter d'entendre des opinions différentes de la sienne, pour que les erreurs et les violations des prescriptions faites par les opérateurs puissent s'exprimer sans que le management use de son pouvoir coercitif. La question dépasse donc le souhait de changer sa politique de sécurité. Elle concerne sa propre capacité à analyser et traiter ses vulnérabilités.

\subsubsection{Identification d'un référent $\mathrm{FHO}$ et des acteurs porteurs de la démarche}

Une condition également très importante est l'identification d'un référent $\mathrm{FHO}$ et des acteurs qui en seront porteurs au sein des équipes. C'est le référent FHO qui, dans un premier temps, apportera des éléments du travail réel, réfléchira au traitement des difficultés de terrain et amènera les acteurs à réfléchir sur leur propre travail. 
L'identification des autres acteurs qui porteront la démarche est également nécessaire. Des appuis à notre démarche ont été trouvés au sein du Codir. Le demandeur était un adjoint du directeur et, plus que cela, il était convaincu du besoin de développer une démarche FHO. L'expert prévention, qui a suivi la recherche depuis son démarrage, est également devenu progressivement persuadé de la nécessité de faire évoluer la politique de sécurité en s'appuyant sur les compétences des opérateurs du terrain. Il a toujours été un interlocuteur important auprès des techniciens du site à travers la diffusion de messages et de pratiques FHO. La capacité à détecter ces catalyseurs, ou les acteurs porteurs de la démarche, est donc une condition considérable.

\subsubsection{Qualité du couplage Référent-Codir}

Au-delà de l'engagement du Codir et de l'identification des acteurs porteurs de la démarche, un travail constant d'articulation entre ces deux groupes est nécessaire. Cette articulation permet aux acteurs des deux groupes de partager un certain nombre d'éléments théoriques et pratiques qui sont fondamentaux pour le bon déroulement de l'intervention. Le développement de la recherche a permis de construire un référentiel théorique partagé entre les chercheurs et les managers grâce à des boucles de régulation constante entre eux. Ces boucles de régulation ont eu lieu via des points téléphoniques, des réunions de restitution à chaque étape de la recherche, et des réunions entre les chercheurs et les managers. Un exemple assez illustratif de ce couplage est la construction du référentiel sécurité 2014-2015 de l'entreprise. Il s'agit d'un document concernant les fondamentaux en sécurité qui a été très influencé par cette recherche et par les avis directs des chercheurs. Il a été construit fin 2013 sur la base d'allers-retours entre les managers et l'équipe de chercheurs. Cet exemple traduit un travail de combinaison entre d'une part, la capacité du Codir à se remettre en cause et son envie de changer, et d'autre part, une offre de l'équipe de chercheurs d'éléments de référentiel et d'une manière de réfléchir qui était en phase avec leurs souhaits.

\subsection{Conditions relatives à la mise en place des EDT}

Les conditions relatives à la mise en place des EDT concernent à la fois des éléments internes et périphériques à l'espace de débat. Ils sont détaillés ci-après.

\subsection{1. À l'intérieur de l'espace de débat : un débat régulier sur le travail réel, finalisé par la recherche de solutions et animé et garanti par le manager de proximité}

52 La première condition du débat est de se centrer sur le travail réel. Pour cela, il doit s'appuyer sur des photos, des vidéos, des récits de cas, etc. dans la mesure du possible, afin d'éviter des discours généraux sur le travail.

53 Une deuxième condition du débat structuré est d'articuler les deux objectifs d'analyse et d'action. Comme l'affirment Mollo \& Nascimento (2013), toute pratique réflexive doit permettre d'analyser la singularité des situations mais doit également avoir pour objectif de développer collectivement des solutions techniques ou organisationnelles. Sans action, l'utilité de l'EDT peut être remise en cause et conduire à son arrêt.

54 Une troisième condition concerne les membres de l'EDT : le débat doit compter sur un socle très régulier pour que chacun puisse suivre les dossiers traités par le collectif et bénéficier $\mathrm{du}$ processus d'apprentissage; et pour qu'il puisse s'approprier 
progressivement l'approche du débat structuré sur le travail, ses principes, ses conditions, ses objectifs.

Si ces trois conditions sont essentielles, l'animation du débat est également une question primordiale. Selon Detchessahar (2013), trois caractéristiques sont nécessaires à développer lors de l'animation du débat : le droit à la prise de parole, le droit à l'erreur et le devoir d'écoute. Le droit à la prise de parole est le droit de s'exprimer sans se le faire reprocher. C'est l'idée d'échanger et de confronter nos représentations avec celles des collègues, qui ne seront pas forcément en accord avec nous. Le droit à l'erreur concerne le fait que l'on peut s'exprimer même sur des faits qui peuvent nous mettre en cause, sans être jugé. Débattre de ces éléments est une occasion d'apprentissage pour le groupe et permet d'éviter que ces situations se répètent sur le terrain. En outre, si le groupe est principalement formé par des opérateurs de terrain, le débat doit être accepté par ces opérateurs. Être accepté signifie qu'ils sont d'accord pour discuter et exposer leurs pratiques, y compris celles qui sont informelles. Le devoir d'écoute, quant à lui, renvoie au fait que l'animateur du débat est dans un esprit d'écoute de tous les participants, pour comprendre ce qu'ils ont à dire et pourquoi ils le disent. C'est le devoir de comprendre les raisons exprimées par les opérateurs pour en tirer des enseignements. À ces conditions d'animation de Detchessahar (2013), on en ajoute une quatrième : il s'agit du devoir de communiquer localement sur le traitement. Cela veut dire que lorsque l'on décide collectivement d'une action à mettre en place pour faire face à telle situation, ou de s'en remettre à d'autres acteurs plus compétents, l'animateur doit faire un retour lors d'une réunion prochaine afin que les agents soient informés des suites données à l'anomalie remontée. Même si pour des raisons diverses, aucune action n'a été engagée, il est important pour les agents d'en être informés et d'en comprendre les raisons (l'absence d'action ne signifiant pas nécessairement une absence de traitement).

L'implication du management de proximité dans l'animation des EDT était également une condition d'expérimentation des EDT au sein de l'entreprise. D'une part, la co-animation chercheur-manager a permis à ce dernier de s'approprier les conditions d'animation citées ci-dessus, et de passer d'une logique descendante à une logique compréhensive. D'autre part, l'animation par le manager garantit la pérennité de la démarche ainsi que la mise en œuvre d'actions d'amélioration.

\subsubsection{Autour de l'espace de débat : le développement de la confiance, le processus de co-construction et le développement du pouvoir d'agir comme éléments clés}

Trois conditions périphériques nous paraissent essentielles pour construire des débats structurés sur le travail: le développement de la confiance des participants, la coconstruction des dispositifs et le développement du pouvoir d'agir local.

La confiance est un préalable à la mise en débat du travail. Au-delà de la confiance que les techniciens nous ont accordée pour expérimenter le dispositif, ils ont gagné en confiance envers le dispositif lui-même au fur et à mesure des séances parce qu'ils ont compris que le but n'était pas de les juger, que certaines situations commençaient à être réglées par les instances supérieures, et parce qu'ils ont eu des retours de la part du management sur les situations qu'ils avaient remontées. Comme le dit Detchessahar (2013), au-delà d'un préalable, la confiance est surtout un produit de la discussion qui se construit lorsque l'on prend l'habitude de se parler du travail. Selon cet auteur, il ne faut pas attendre d'avoir 
supprimé la méfiance pour construire la confiance, mais commencer à ouvrir les espaces de discussion pour construire la confiance.

La co-construction et l'adaptation locale des dispositifs de débat sont également une condition essentielle. Le dispositif construit a connu des adaptations particulières afin de répondre aux spécificités de l'organisation locale du site travaillé. La remontée des situations via des photos, la composition du groupe (des techniciens avec leur manager), étaient un choix collectif des participants. Cette adaptation locale nous apparaît essentielle dans la mesure où elle permet d'intégrer un dispositif nouveau via la transformation de l'organisation actuelle et non via l'ajout d'un projet dans une organisation existante, ce qui n'engendre pas un coût/travail supplémentaire pour les personnes. Tout comme les dispositifs locaux de mise en débat, le dispositif systémique a subi des adaptations locales en fonction de l'organisation des groupes. La remontée via le CPS, puis au niveau de la réunion du Codir étaient des décisions collectives prises avec tous les participants. Ce caractère adaptable était fondamental pour que, localement, le dispositif soit effectif et durable. Si l'on change de site ou d'organisation, il faut que le dispositif systémique soit réadapté avec les agents de ce nouveau site.

Enfin, la question de fond relative au traitement des situations de terrain remontées est liée au principe de la subsidiarité : quel est le niveau pertinent le plus bas pour gérer la situation discutée? En écho à cette question, l'approche développée en sous-entendait une autre : que doit faire le niveau supérieur pour donner du pouvoir d'agir au niveau inférieur afin que ce dernier ait les ressources suffisantes pour traiter les questions remontées ? Ces questions ont été travaillées avec les niveaux supérieurs et ont permis de développer le pouvoir d'agir local. Lorsqu'une situation arrivait à l'expert prévention et qu'il s'occupait, par exemple, de modifier une règle pour l'adapter à la réalité du terrain, ou lorsque l'expert prévention fournissait au site 4 ou 5 modèles différents de portes de coffret pour que localement les techniciens puissent régler cette situation sans forcément la remonter au secteur de la prévention, les agents de terrain gagnent de l'autonomie et du pouvoir d'agir qu'ils n'avaient pas auparavant pour mettre en place des actions. L'objectif du débat structuré sur le travail n'est donc pas d'augmenter les situations remontées à d'autres niveaux (au risque de saturer leur capacité de traitement), mais plutôt d'en avoir le moins possible, en donnant aux niveaux inférieurs les moyens suffisants pour les traiter. Sachant que le principe de la subsidiarité renvoie à la «recherche permanente du niveau le plus pertinent pour l'action» (Petit, Dugué, \& Daniellou, 2011, p. 404), l'idée était donc de réfléchir, à chaque situation remontée et discutée, au développement de ressources aux niveaux inférieurs pour leur traitement.

\section{Le développement d'un environnement capacitant}

61 Comme les résultats le montrent, la réflexion collective conduite dans l'EDT est de plus en plus tournée vers les situations futures probables ou l'anticipation de situations à risque. Les agents sont donc rentrés progressivement dans une dynamique d'analyse, d'anticipation et de traitement qui a permis de changer le type de gestion de la sécurité développée dans l'entreprise. Désormais le collectif discute, anticipe les risques possibles et traite les sujets ensemble, dans une logique non plus seulement de correction mais aussi de prévention. Quant aux situations remontées par les salariés, elles ne sont plus motivées par une logique du chiffre (répondre aux objectifs managériaux), mais par la 
volonté de chacun de participer à l'analyse et au traitement collectif de la situation exposée, et de contribuer à la mise en place d'actions d'amélioration.

Cette recherche a également permis de développer la confrontation de perceptions individuelles via le débat sur le travail. La subsidiarité a été organisée de manière que les situations qui ne peuvent pas être gérées localement le soient à d'autres niveaux de l'organisation, jusqu'au Codir ou même au niveau national. Falzon (2013) définit un « environnement capacitant» comme celui qui permet aux personnes de développer de nouvelles compétences et connaissances, d'élargir leurs possibilités d'action, leur degré de contrôle sur leur tâche et sur la manière dont ils la réalisent. Il s'agit donc d'un environnement qui favorise l'autonomie et le pouvoir d'agir des travailleurs.

Plusieurs éléments présentés dans cet article conduisent à conclure que la rechercheaction a permis de développer un environnement capacitant.

Premièrement, la recherche a contribué au développement d'une organisation fondée sur la confrontation des points de vue à travers le débat de situations de travail quotidiennes. Le débat a été organisé en tenant compte du principe de subsidiarité, de manière que les situations qui ne peuvent pas être gérées localement le soient à d'autres niveaux de l'organisation, jusqu'au Codir ou même au niveau national. La conséquence est une meilleure connaissance du terrain des niveaux hiérarchiques supérieurs, et la mise en œuvre d'actions d'amélioration qui ne se limitent pas au niveau de la sécurité mais qui englobent aussi la qualité du travail, la performance du système et la santé des travailleurs. L'organisation progresse ainsi à partir de sa propre expérience : certaines règles et procédures ont été reformulées, des décisions stratégiques ont été prises, toutes en fonction des données du terrain. Par ailleurs, l'organisation de la subsidiarité a redonné du pouvoir local au niveau des sites et ce faisant, a développé le pouvoir d'agir des techniciens et du manager de proximité. En effet, les situations de travail remontées par les salariés ne sont plus motivées par une simple logique de reporting (selon laquelle les sites jouaient un rôle de " chambre d'enregistrement »), mais par la volonté de chacun de participer à l'analyse et au traitement collectif des situations exposées. La régularité et l'utilité des débats (mise en œuvre d'actions concrètes) les encouragent à maintenir le dispositif; ils sont de plus en plus plus motivés pour prendre des photos, les mettre en débat et décider des actions de gestion.

Deuxièmement, la dynamique du débat est de plus en plus tournée vers les situations futures probables ou l'anticipation de situations à risque. Les agents sont donc rentrés progressivement dans une dynamique d'analyse, d'anticipation et de traitement qui a permis de changer le type de gestion de la sécurité développée dans l'entreprise. Désormais le collectif discute, anticipe les risques possibles et traite les sujets ensemble, dans une logique non plus seulement de correction mais aussi de prévention.

Troisièmement, l'EDT participe à l'apprentissage mutuel et à l'intégration des nouveaux. D'un côté, les plus expérimentés sont valorisés puisqu'ils mettent en partage les connaissances et compétences acquises pendant de nombreuses années de travail. D'un autre côté, les plus jeunes sont dans une dynamique d'apprentissage continu, en discutant de situations d'urgence et de dépannage qu'ils n'ont pas forcément appris en formation ou vécus sur le terrain. Cela permet de prévenir l'exclusion et d'encourager l'intégration sociale. Par ailleurs, l'apprentissage en continu permet à la fois aux jeunes et aux plus expérimentés d'être à jour de l'évolution des processus techniques et des matériels. 
Tous ces éléments montrent que la sécurité telle qu'elle est organisée par le débat structuré sur le travail, n'est plus vécue comme un état figé à un moment donné, mais plutôt comme un apprentissage collectif permanent de l'expérience passée à travers le débat actuel pour prévenir des risques futurs. Le rôle du collectif est maintenant déterminant dans l'acceptabilité des risques par le groupe et, ainsi, dans les actions qui protègent ou exposent le système. Nous pouvons penser également que cette approche a pu contribuer au développement d'une "culture juste» (Groupe de travail de l'Icsi "Culture de sécurité », 2017), c'est-à-dire, la mise en place d'un processus organisationnel de questionnement systématique permettant, lorsqu'un événement non souhaitable s'est produit, de définir de façon partagée la limite entre l'acceptable et l'inacceptable et de supprimer la peur du blâme. Il s'agit d'un contexte où le collectif de travail est capable de reconnaître les initiatives positives contribuant à la sécurité (signalement, contribution à l'amélioration continue, soutien à des collègues en difficulté...) ainsi que les comportements non acceptables (par exemple, le fait de fumer dans des zones non permises d'une entreprise de distribution de gaz).

\section{Conclusion}

68 Cet article décrit et présente les bénéfices du « débat structuré sur le travail », qui repose sur deux piliers : le développement des espaces de débat sur le travail et l'organisation de la subsidiarité. Si certaines conditions sont respectées, le débat structuré sur le travail peut favoriser le déploiement d'un système qui permet un apprentissage en continu et développe les capacités des individus et de l'organisation à faire face à la variabilité, et ainsi à prévenir les risques.

La recherche présentée se heurte cependant à quelques limites et difficultés qui ouvrent de nouvelles perspectives de recherche. D'une part, l'expérimentation a été conduite sur un seul site. Bien que la généralisation de la démarche ait été travaillée à travers des formations-actions et un suivi auprès de quelques managers, il est nécessaire de développer le débat structuré sur le travail sur d'autres sites pour approfondir la réflexion sur les conditions de leur mise en place. D'autre part, la démarche engagée demeure encore très liée à des personnes. Si ces personnes quittent l'entreprise, et si les conditions de mise en place du débat structuré sur le travail ne sont pas respectées, le dispositif peut se fragiliser. Notons cependant qu'à ce jour la dynamique engagée se poursuit, et qu'un expert FHO a été embauché au sein de l'entreprise pour en assurer la pérennité. Enfin, le dispositif de mise en débat a été organisé de manière à articuler logique descendante («top down») et ascendante ("bottom up») selon le principe de subsidiarité. Une perspective de poursuite intéressante serait de travailler la transversalité des secteurs, c'est-à-dire d'organiser des EDT entre managers pour traiter de problématiques transversales communes à deux ou plusieurs services. Nous faisons l'hypothèse que ce type de fonctionnement transversal non seulement permettrait de progresser encore plus sur les questions de sécurité par le partage et l'expérimentation de solutions communes, mais serait également un outil de prévention pour la santé des managers.

Quoi qu'il en soit, la manière de conduire l'intervention a produit des connaissances, des effets pérennes sur l'organisation et a contribué à des développements ultérieurs. Or, il est maintenant admis que la recherche en ergonomie vise à produire non pas seulement des modèles de l'activité des travailleurs observés, mais aussi des modèles de l'action 
ergonomique de transformation (Petit, Querelle, \& Daniellou, 2007). Dans ce sens, les transformations opérées au travers de cette recherche-action sont le produit d'une intervention elle-même capacitante, censée produire des nouvelles connaissances et compétences pour l'ergonomie en tant que discipline scientifique. Dans ce cas, «l'ergonome sera sans doute mieux armé-e pour comprendre et prendre en compte les débats de normes à l'œuvre chez ses interlocuteurs, et pour développer des interventions [encore] plus pertinentes » (Daniellou, 2006, p. 16).

\section{BIBLIOGRAPHIE}

Amalberti, R. (2013). Piloter la sécurité, Paris : Springer.

Arnoud, J. (2013). Conception organisationnelle : pour des interventions capacitantes. Thèse de doctorat en ergonomie. CNAM, Paris, France.

Barcellini, F. (2015). Développer des interventions capacitantes en conduite du changement. Comprendre le travail collectif de conception, agir sur la conception collective du travail. Habilitation à Diriger des Recherches. Université de Bordeaux.

Conlon, D. E., Meyer, C.J., \& Nowakowski, J.M. (2005), How Does Organizational Justice Affect Performance, Withdrawal, and Counterproductive Behavior? In J. Greenberg \& J.A. Colquitt (Eds.), Handbook of Organizational Justice (pp. 301-327). Mahwah : Lawrence Erlbaum Associates.

Daniellou, F. (2006). Entre expérimentation réglée et expérience vécue : Les dimensions subjectives de l'activité de l'ergonome en intervention. Activités, 3(1), 5-18, http:// www.activites.org/v3n1/daniellou.pdf

Daniellou, F. (2013). L'ergonome et les débats sur la performance de l'entreprise. In J. Petit, K. Chassaing, \& S. Aubert (Eds.), Des pratiques en réflexion (pp 225-240). Toulouse : Octarès Editions.

Daniellou, F., \& Garrigou, A. (1993). L'ergonome, l'activité, et la parole des travailleurs. In : A. Weill-Fassina, P. Rabardel, \& D. Dubois (Eds.), Représentations pour l'action (pp. 73-92). Toulouse : Octarès.

Daniellou, F., Simard, M., \& Boissières, I. (2010). Facteurs humains et organisationnels de la sécurité industrielle : un état de l'art. Numéro 2010-02 des Cahiers de la Sécurité Industrielle, Fondation pour une Culture de Sécurité Industrielle, Toulouse, France (ISSN 2100-3874). Disponible à l'URL http://www.FonCSI.org/fr/cahiers/ (Dernier accès le 06 août 2014).

Dejours, C. (1998). Souffrance en France. La banalisation de l'injustice sociale. Paris : Seuil.

Detchessahar, M. (2001). Quand discuter, c'est produire... Pour une théorie de l'espace de discussion en situation de gestion. Revue française de gestion, 132, 32-43.

Detchessahar, M. (2011). Quand le management n'est pas le problème mais la solution..., Revue française de gestion, 5(214), 89-105.

Detchessahar, M. (2013). Faire face aux risques psycho-sociaux : quelques éléments d'un management par la discussion. Négociations, 19(1), 57-80. 
Detchessahar, M., \& Journé, B. (2007). Une approche narrative des outils de gestion. Revue Française de Gestion, 33(174), 77-92.

Falzon, P. (2013). Ergonomie Constructive. Paris : PUF.

Falzon, P., \& Mollo, V. (2009). Pour une ergonomie constructive : les conditions d'un travail capacitant. Laboreal, 5(1), 61-69.

Fauquet, P. (2006). Confrontation croisée ou analyse collective sur la base de restitutions d'entretiens individuelles : deux approches pour l'analyse évènementielle. Activités, 3(2), 2-14, http://www.activites.org/v3n2/activites-v3n2.pdf

Greenberg, J., \& Edwards, M. (2009). Voice and silence in organizations. Bingley, UK : Emerald Publishing Group.

Groupe de travail de l'Icsi «Culture de sécurité » (2017). La culture de sécurité : comprendre pour agir. D. Besnard, I. Boissières, F. Daniellou, J. Villena (Eds.). Numéro 2017-01 de la collection les Cahiers de la sécurité industrielle, Institut pour une culture de sécurité industrielle, Toulouse, France.

Kerguelen, A. (2003). Actogram Kronos pour Windows. Toulouse : Éditions Octarès.

Linhart, D. (1991). Le torticolis de l'autruche ; l'éternelle modernisation des entreprises. Paris : Seuil.

Linhart, D. (2004). La modernisation des entreprises. Paris : La Découverte.

Llory, M. (1996). Accidents industriels, le coût du silence. Opérateurs privés de parole et cadres introuvables. Paris : L'Harmattan.

Millon-Delsol, C. (1993). Le Principe de subsidiarité. Paris : PUF, coll. « Que sais-je ?».

Mollo, V., \& Nascimento, A. (2013). Pratiques réflexives et développement des individus, des collectifs et des organisations. In P. Falzon (Ed.), Ergonomie constructive (pp. 164-175). Paris : PUF.

Morrison, E., \& Milliken, F. (2000). Organizational Silence: a barrier to change and development in a pluralistic world. The Academy of Management Review, 25(4), 706-725.

Petit, J., Dugué, B., \& Daniellou, F. (2011). L'intervention ergonomique sur les risques psychosociaux dans les organisations : enjeux théoriques et méthodologiques. Le Travail Humain, 74(4), 391-409.

Petit, J., Querelle, L., \& Daniellou, F. (2007). Quelles données pour la recherche sur la pratique de l'ergonome. Le Travail Humain, 70(4), 391-411.

Pinder, C., \& Harlos, P. (2001). Employee Silence: Quiescence and Acquiescence as Responses to Perceived Injustice. In G.R. Ferris (Ed.), Research in Personnel and Human Resources Management, vol. 20 (pp. 331-369). Greenwich : JAI Press.

Pradines, P. (2004). Management : la subsidiarité, organisation de l'entreprise et enseignement de l'Église, http://biblio.domuni.org/articlesphilo/subsidiarite/

Rocha, R. (2014). Du silence organisationnel au développement du débat structuré sur le travail : les effets sur la sécurité et sur l'organisation. Thèse de Doctorat en Ergonomie. Université de Bordeaux.

Rocha, R., Mollo, V., \& Daniellou, F. (2015). Work debate spaces: a tool for developing a participatory safety management. Applied Ergonomics, 46, 107-114.

Tangirala, S., \& Ramanujan, R. (2008). Employee Silence on Critical Work Issues: The Cross Level Effects of Procedural Justice Climate. Personnel Psychology, 61(1), 37-68. 
Vakola, M., \& Bouradas, D. (2005). Antecedents and Consequences of Organisational Silence: An Empirical Investigation. Employee Relations, 27(5), 441-458.

Van Belleghem, L., \& Forciolli Conti, E. (2015). Une ingénierie de la discussion ? Chiche! In Actes du $50^{\text {ème }}$ congrès de la SELF. Paris, 23 au 25 septembre.

Van Dyne, L., Ang, S., \& Botero, I.C. (2003). Conceptualizing Employee Silence and Employee Voice as Multidimensional Constructs. Journal of Management Studies, 40(6), 1359-1392.

\section{NOTES}

1. Nous avons fait le choix pour cet article de décrire le plus exhaustivement possible l'organisation d'espaces de débat sur le travail au sein de l'entreprise. Il ne nous est donc pas possible, pour des raisons de place, de décrire aussi finement les méthodes d'analyse des données recueillies via ces espaces. Le lecteur intéressé pourra se référer à Rocha (2014) pour plus de détails.

2. Les premiers articles s'intéressant au thème sont apparus au début des années 2000 avec Morrison et Milliken (2000) et Pinder et Harlos (2001). En 2003, le «Journal of Management Studies" devient la $1^{\text {re }}$ revue dédiée entièrement au silence dans les organisations. Et en 2009 , «Voice and Silence in Organizations» est le premier livre s'intéressant spécifiquement à ce thème (Greenberg \& Edwards, 2009).

3. Pour une présentaillée détaillée du diagnostic, voir Rocha (2014)

4. Pour plus de détails concernant la méthode d'analyse des données, voir Rocha (2014).

5. Le terme événement significatif est utilisé dans les centrales nucléaires d'EDF pour signifier des écarts par rapport au référentiel prescriptif (Fauquet, 2006). On s'est approprié ce terme pour les dispositifs de débat conçus.

6. Pour connaître d'autres exemples, des différents niveaux de boucles de traitement, voir Rocha (2014).

\section{RÉSUMÉS}

Cet article est issu d'une recherche-action conduite dans le champ de la sécurité des organisations, au sein d'une entreprise de distribution d'électricité. Une première phase de diagnostic a montré l'existence d'un « silence organisationnel » : les travailleurs sont conduits à ne plus remonter les difficultés vécues sur le terrain, ou à remonter des situations à faible impact. Pour lutter contre ce silence organisationnel, une seconde phase a consisté à développer le « débat structuré sur le travail ", c'est-à-dire des espaces de débat organisés par le principe de subsidiarité à différents échelons de l'entreprise. Dès que certaines conditions sont respectées, ces espaces peuvent contribuer au développement des salariés et de l'organisation elle-même. Dans le même temps, le débat structuré sur le travail permet de développer un environnement plus capacitant, c'est-à-dire un environnement qui fournit les moyens du développement de l'activité. L'objectif principal de cet article est de présenter les contributions et les conditions d'une telle approche et de montrer à quel point elle peut contribuer au développement d'un environnement capacitant. 
This article is based on a doctoral thesis inscribed in the field of the security of organizations. The research was carried out in an electricity distribution company subject to "organizational silence", where workers are required to no longer report difficulties experienced on the ground or situations with low impact. This research defends the idea that in order to deal with organizational silence, it is necessary to develop the "structured debate on work" - i.e. spaces of debate organized by the principle of subsidiarity - at different levels of the company. As soon as certain conditions have been met, these spaces can contribute to the development of the persons concerned and of the organization itself. At the same time, the structured debate on work makes it possible to develop a more capable environment, that is to say an environment which provides the means to develop the activity. The main objective of this article is to present the contributions and conditions of this approach and show how well it can develop an enabling environment.

\section{INDEX}

Mots-clés : silence organisationnel, débat sur le travail, subsidiarité, organisations capacitantes

Keywords : organizational silence, work debate, subsidiarity, learning organisations

\section{AUTEURS}

\section{RAONI ROCHA}

Université Fédérale d'Itajubá, Campus Itabira, Itabira, MG, Brésil. roani.france@gmail.com

\section{VANINA MOLLO}

Université de Toulouse, INPT, IPST-Cnam, CERTOP (UMR5044), Toulouse. vanina.mollo@ipstcnam.fr

\section{FRANÇOIS DANIELLOU}

Directeur scientifique de la Fondation pour une culture de sécurité industrielle (Foncsi), Toulouse. francois.daniellou@fonsci.icsi-eu.org 